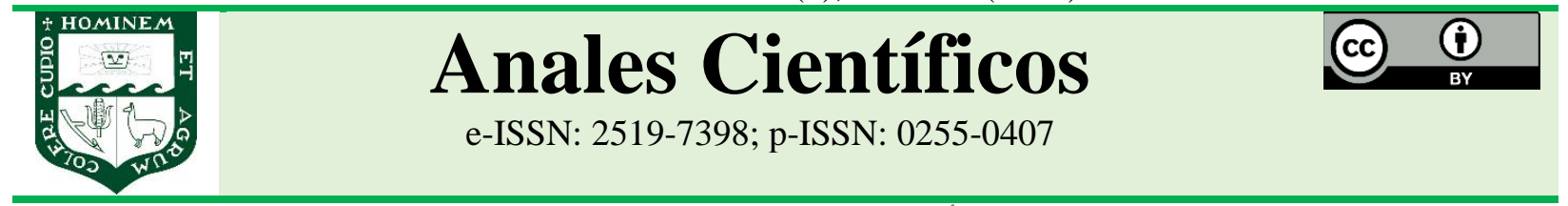

ARTÍCULO ORIGINAL - RESEARCH ARTICLE

http://dx.doi.org/10.21704/ac.v82i2.1789

\title{
MASCULINIZACIÓN DE ALEVINOS DE TILAPIA DEL NILO (Oreochromis niloticus) CON EL INHIBIDOR DE AROMATASA LETROZOL
}

\section{Masculinization of Nile tilapia fry (Oreochromis niloticus) with the aromatase inhibitor letrozole}

\author{
Elsa Vega Galarza ${ }^{1 *(D)}$; Nieves Sandoval Chaupe ${ }^{2}$ iD ; Beatriz Elena Angeles Escobar ${ }^{1}$ \\ ${ }^{1}$ Facultad de Pesquería, Universidad Nacional Agraria La Molina, Lima15024, Perú. \\ ${ }^{2}$ Laboratorio de Ictiopatología, Universidad Nacional Mayor de San Marcos, Lima, Perú. \\ *evegalarza@1amolina.edu.pe
}

Recibido: 20/09/2021; Aceptado: 01/11/2021; Publicado: 31/12/2021

\begin{abstract}
This paper presents the results of two experiments for the masculinization of tilapia fry (Oreochromis niloticus) through the use of an aromatase inhibitor, letrozole, administered in fish feed. The first experiment evaluated the effect on growth, survival, and the percentage of masculinization of sexually undifferentiated fish undergoing letrozole doses of 50 (T1), 100 (T2), and 200 (T3) $\mathrm{mg} \mathrm{kg}^{-1}$ during four weeks. There was a negative control group (C0) feed without letrozole and a positive control group (C) that received $60 \mathrm{mg} \mathrm{kg}^{-1}$ of 17 alpha-methyltestosterone (MT) in feed. No significant differences in growth (weight and length) and survival were found between treatments and control groups. Treatments T1, T2, T3 showed a percentage of 90\%, 100\% and 93\% of males, not showing significant differences. It was found $66 \%$ of males in group $\mathrm{C}$ and $45 \%$ in group $\mathrm{C} 0$, showing significant differences between each other and with respect to all letrozole treatments. It was found 3\% of females in $\mathrm{T} 1$ and $7 \%$ of fish with intersex gonads in both $\mathrm{T} 1$ and $\mathrm{T} 3$. In the second experiment, the fishes were feed a dose of $50 \mathrm{mg} \mathrm{kg}^{-1}$ of letrozole during two weeks $(2 \mathrm{~S})$ and three weeks $(3 \mathrm{~S})$. The results were $73 \%$ of males for $2 \mathrm{~S}$ and $82 \%$ for $3 \mathrm{~S}$, showing significant differences. It is concluded that letrozole in doses between 50 and $200 \mathrm{mg} \mathrm{kg}^{-1}$ achieves the masculinization of more than $90 \%$ of the $O$. niloticus fry, after 4 weeks of treatment without affecting survival and growth.
\end{abstract}

Keywords. aromatase inhibitor | letrozole | masculinization | sex inversion | tilapia

\section{RESUMEN}

Se presentan los resultados de dos experimentos para la masculinización de alevinos de tilapia (Oreochromis niloticus) con un inhibidor de aromatasa, letrozol, administrado en el alimento. En el primer experimento se evaluó el efecto sobre el crecimiento, la supervivencia y el porcentaje de masculinización de peces no diferenciados sexualmente, sometidos a dosis de letrozol de 50 (T1), 100 (T2) y 200 (T3) $\mathrm{mg} \mathrm{kg}^{-1}$ en el alimento durante cuatro semanas. Un control negativo (C0) recibió alimento sin letrozol y un control positivo (C) recibió alimento con 60 $\mathrm{mg} \mathrm{kg}^{-1}$ de $17 \alpha$ metiltestosterona (MT). No hubo diferencias significativas entre tratamientos y controles en el crecimiento (en longitud y peso) y la supervivencia. Los porcentajes de masculinización fueron $90 \%, 100 \%$ y $93 \%$ 
Vega, E., Sandoval, N., \& Angeles, B. (2021). Anales Científicos. 82(2), 262-278. DOI. 10.21704/ac.v82i2.1789

en T1, T2 y T3 respectivamente sin diferencias significativas. La masculinización en el grupo C fue $66 \%$ y en el grupo C0 fue 45\%, mostrando diferencias significativas entre sí y también respecto a los 3 tratamientos con letrozol. Se encontró 3\% de hembras en T1 y 7\% de peces con gónadas intersexo tanto en T1 como en T3. En el segundo experimento, se suministró una dosis de $50 \mathrm{mg} \mathrm{kg}^{-1}$ de letrozol durante dos semanas (2S) y tres semanas (3S). Los resultados dieron $73 \%$ de machos para $2 \mathrm{~S}$ y $82 \%$ para $3 \mathrm{~S}$ con diferencias significativas. Se concluyó que el letrozol en dosis entre 50 y $200 \mathrm{mg} \mathrm{kg}^{-1}$ logró más del $90 \%$ de machos en 4 semanas sin afectar la supervivencia y el crecimiento.

Palabras clave: inhibidor de aromatasa | inversión sexual | letrozol | masculinización | tilapia

Forma de citar el artículo (Formato APA):

Vega, E., Sandoval, N., \& Angeles, B. (2021). Masculinización de alevinos de tilapia del Nilo (Oreochromis niloticus) con el inhibidor de aromatasa letrozol. Anales Científicos. 81(2), 262-278. http://dx.doi.org/10.21704/ac.v82i2.1789

Autor de correspondencia (*): Elsa Victoria Vega Galarza. Email: evegalarza@lamolina.edu.pe

(C) Los autores. Publicado por la Universidad Nacional Agraria La Molina.

This is an open access article under the CC BY

\section{INTRODUCCIÓN}

El cultivo de la tilapia a nivel mundial es realizado principalmente con individuos machos debido a que éstos presentan un mayor crecimiento, obteniéndose una mayor uniformidad de tamaños y un mejor control de la reproducción que no es posible realizar en cultivos con ambos sexos (Beardmore et al., 2000, El-Graizy y El-Gamal, 2012). La técnica disponible comercialmente para producir alevinos machos es la de inversión sexual mediante la administración de la hormona $17 \alpha$ metiltestosterona (MT) en el alimento, antes de la diferenciación sexual de los peces, con resultados mayores al 90 por ciento de individuos machos. Las dosis por $\mathrm{kg}$ de alimento utilizadas varían de un autor a otro, sin embargo, el rango más frecuentemente reportado es de 40 a $60 \mathrm{mg}$ y tratamientos de 4 semanas (Drummont et al., 2009; Ferdous y Ali, 2011; Klipp et al., 2019; MainardesPinto et al., 2000, Popma y Green, 1990). Existen otros factores que afectan el porcentaje de machos esperado con el tratamiento de MT, como la disminución de estabilidad de la hormona con el tiempo y temperatura durante el almacenamiento del alimento preparado (Barry et al., 2007, Teichert-Coddington et al., 2000), la presencia de fitoestrógenos en los insumos proteicos vegetales para la elaboración del alimento (El-Sayed et al., 2012), el establecimiento de jerarquías entre los peces durante el tratamiento "afectando el consumo del alimento y la cantidad de hormona que ingiere cada pez", la longitud de los peces al inicio del tratamiento, la tasa y frecuencia de alimentación, la temperatura y el sistema de cultivo (Drummont et al., 2009, Hiott y Phelps, 1993, Klipp et al., 2019, Meurer et al., 2012). Las diversas prácticas de manejo durante la producción de alevinos de tilapia pueden explicar los diferentes resultados obtenidos en los centros de producción, afectando la calidad de la semilla disponible en el mercado nacional.

Respecto a los riesgos asociados al uso de la MT en acuicultura, algunos estudios realizados concluyeron que no hay riesgo para el consumidor ni para los acuicultores (Green y Teichert-Coddington, 2000; Macintosh, 2010, Zanardi et al., 2011), años después Mlalila et al. (2015) indican que la cantidad de hormona utilizada es mayor a la requerida para la inversión sexual por lo que los peligros ambientales deben ser enfatizados, Thanasupsin et al. (2021) mencionan que existe riesgo ecológico en las descargas de agua de los estanques de masculinización con MT, pudiendo causar un efecto adverso en el rendimiento reproductivo de los peces evaluados en su estudio (Probarbus jullieni, Barbonymus gonionotus y Anabas testudineus), en otro trabajo sobre los niveles de toxicidad de la hormona en Danio rerio se concluye que la MT tiene un potencial tóxico para los peces porque altera las vías metabólicas enzimáticas y puede suponer un riesgo para los ecosistemas (Rivero-Went et al., 2020). La producción de tilapia en nuestro país es de 3099 t en el 2020 (Ministerio de la Producción, 2021) y tiene una proyección optimista de crecimiento para el 2025 de 
27000 t (Baltazar et al., 2018) proyectándose asimismo la producción de alevinos, por lo que se hace necesario considerar los peligros ambientales y riesgos ecológicos de la actividad.

En busca de otras formas de producir alevinos machos, diversos estudios han sido realizados para evaluar otros métodos de masculinización para la producción comercial de alevinos, entre ellos métodos directos e indirectos, uso de anti estrógenos, inhibidores enzimáticos, sustancias esterilizantes, feminización de machos para la producción de super machos, hibridación, manipulación de factores ambientales, etc., sin embargo, pocos han logrado resultados como los de la masculinización mediante la hormona MT, la cual permite una producción comercial de forma extendida (Bombardelli et al., 2004).

El uso de inhibidores enzimáticos como es el caso de los inhibidores de la aromatasa (IA) ha sido reportado en numerosos estudios relacionados a la investigación de la actividad del complejo citocromo P450 aromatasa (P450arom) y su importancia en la diferenciación gonadal de los peces; con resultados que confirman su influencia en la inversión del sexo de hembras a machos durante el proceso de diferenciación de las gónadas en desarrollo, reduciendo la producción de estrógenos (Steele et al., 1987 citados por Gao et al., 2010), sin embargo aún no es factible aplicarlos a escala comercial debido a la falta de protocolos que definan dosis efectivas, tiempos de administración, efectos residuales en los peces e impacto sobre el medio ambiente, así como costos de tratamiento mayores a los ya usados comercialmente. Inhibidores como el exemestano, fadrozole y el letrozol han sido probados en diversos peces y otros vertebrados (Gao et al., 2010; Katare et al., 2015; Shen et al., 2015). El letrozol esta disponible en el mercado nacional y es utilizado en el tratamiento de cáncer de mama estrógeno dependiente. La literatura indica que su empleo en la inversión sexual en tilapias está referida a la tilapia roja y la tilapia mosambica con dosis que varían desde 25 a $200 \mathrm{mg} \mathrm{kg}^{-1}$ de alimento, los resultados en la obtención de individuos machos son variables principalmente en relación al incremento de la dosis, el tiempo de tratamiento y la forma de administración (Betancur et al., 2009; Das et al.; 2012).

En este contexto el trabajo tuvo como objetivo determinar la dosis del inhibidor de la enzima aromatasa (IA) letrozol, en el tratamiento oral para lograr la mayor proporción de alevines machos de tilapia Oreochromis niloticus durante la etapa de diferenciación sexual y su efecto en la supervivencia y el crecimiento. Así como, evaluar la masculinización en diferentes tiempos de tratamiento.

\section{MATERIALES Y MÉTODOS}

Lugar y duración. La investigación fue realizada en el Laboratorio de Acuicultura de la Facultad de Pesquería de la Universidad Nacional Agraria La Molina, tuvo una duración de 8 meses. Se desarrollaron dos etapas experimentales cada una con 4 meses de duración.

Unidades experimentales. En la primera etapa se emplearon 15 acuarios de 60 litros de volumen (tres tratamientos, un control positivo y un control negativo con tres repeticiones cada uno) los cuales fueron lavados y desinfectados con una solución de ácido clorhídrico al $10 \%$, enjuagados profusamente, y se llenaron con 40 litros de agua de pozo mantenida a $28^{\circ} \mathrm{C}$ mediante calefactores con termostato. La aireación fue administrada mediante un sistema compuesto por un blower regenerativo de 1/3 HP (Sweetwater, Aquatic Eco-Systems, Inc, USA), manguerillas de aireación, válvulas reguladoras y piedras difusoras. Se cubrieron tres lados de los acuarios con plástico negro para uniformizar la incidencia de la luz, se trabajó con el fotoperiodo natural. En la segunda etapa se usaron 6 acuarios que fueron asignados para dos tratamientos con tres repeticiones con las mismas condiciones descritas para el primer experimento.

Animales experimentales. En la primera etapa se utilizaron larvas provenientes del desove de reproductores de Oreochromis niloticus del Centro de Investigación Piscícola de la UNALM, fueron trasladadas al Laboratorio en tinas de plástico hasta que completen la reabsorción del saco vitelino, mantenidas con aireación constante y temperatura de $28^{\circ} \mathrm{C}$. Luego se seleccionaron 1950 peces (4-5 días poseclosión) con una longitud total promedio de $9,2 \mathrm{~mm}$ y peso promedio de 0,0075 g. Para la segunda etapa se realizó un procedimiento similar y se seleccionaron 780 peces (56 días poseclosión) con una longitud total de $9,9 \mathrm{~mm}$ y un peso promedio de $0,0127 \mathrm{~g}$. 
Dietas experimentales. Se utilizó un alimento comercial de inicio para tilapias marca Aquatech con 45 $\%$ de proteína y un tamaño de grano de $1.5 \mathrm{~mm}$. El alimento fue molido y tamizado para obtener un tamaño de partícula de $500 \mu \mathrm{m}$. Asimismo, se utilizó alcohol etílico de $96 \%$ v/v como disolvente del letrozol (FEMARA®) y de la hormona MT. Se prepararon 5 dietas experimentales: Alimento con tres concentraciones de letrozol, 50, 100 y $200 \mathrm{mg} \mathrm{kg}^{-1}$ alimento, disolviendo el letrozol en alcohol con cada una de las concentraciones elegidas, luego cada solución fue adicionada en una proporción alimento: alcohol de 2:1, mezclándose homogéneamente para asegurar que todo el alimento quede impregnado del inhibidor. El alimento para el control positivo fue preparado disolviendo en alcohol la hormona 17 alfa metiltestosterona en una concentración de $60 \mathrm{mg}$ por $\mathrm{kg}$ de alimento, la solución fue distribuida de forma similar que en la preparación anterior. El alimento para el control negativo fue preparado sólo con alcohol bajo las mismas proporciones y procedimiento de mezcla descritos. La mezcla de cada una de las dietas fue realizada manualmente utilizando guantes de goma y mascarillas tapa boca. Para el secado de los alimentos estos fueron extendidos en una capa fina permitiendo la evaporación del alcohol durante 24 horas bajo sombra y en un lugar ventilado. Los alimentos completamente secos fueron almacenados en recipientes oscuros debidamente etiquetados en refrigeración hasta su uso. Para la segunda etapa se preparó el alimento con la dosis de $50 \mathrm{mg} \mathrm{kg}^{-1}$ bajo las mismas consideraciones anteriores.

Parámetros ambientales. En ambas etapas experimentales la calidad del agua fue mantenida mediante recambios diarios del 10 hasta el 50 por ciento del volumen total, aumentando el recambio con el incremento de la biomasa en el tiempo. Se retiraron dos veces al día las heces y restos de alimento del fondo de los acuarios. Una vez por semana durante los controles biométricos, los acuarios fueron limpiados en su totalidad con una renovación completa del agua. El agua utilizada en los recambios fue mantenida a la misma temperatura experimental para evitar cambios bruscos. El oxígeno disuelto y la temperatura fueron medidos diariamente durante la mañana (8:00 horas) y la tarde (15:00 horas) con un medidor de oxígeno con sensor de temperatura (modelo 550A, YSI Incorporated. USA) y el pH en las tardes (15:00) con un medidor portátil modelo pHTestr 20 (OAKTON Instruments, USA).

Etapas Experimentales. La investigación fue realizada en dos etapas, en la primera se evaluó tres dosis de letrozol y la segunda se evaluó el efecto de la mejor dosis en tratamientos con diferentes tiempos de aplicación.

Primera etapa: Asignados los tratamientos al azar en las unidades experimentales, los peces fueron sembrados en cada acuario a una densidad de 3000 peces. $\mathrm{m}^{-3}$ (130 por acuario). Durante 4 semanas fueron alimentados según la tabla recomendada por Popma y Green (1990). La primera semana se suministró el alimento a una tasa del $20 \%$ de la biomasa al día, distribuido en cuatro raciones diarias, semanalmente la tasa fue reajustada considerando el incremento de la biomasa ( $g$ ) y la mortalidad en cada unidad experimental, terminando la última semana con una tasa del $15 \%$. Los controles de peso y longitud total se hicieron semanalmente pesando a todos los peces de cada acuario, utilizando una balanza analítica $(0,0001 \mathrm{~g}$ de precisión) marca DENVER, la longitud total fue medida a un 10 por ciento de los peces de cada unidad experimental con una regla graduada en milímetros.

Segunda etapa: Para iniciar el experimento se consideraron los resultados de masculinización de la primera etapa y se seleccionó la dosis de letrozol de 50 $\mathrm{mg} \mathrm{kg}^{-1}$ por su eficiencia en la obtención de individuos machos y por ser la mínima dosis evaluada. De forma similar a la primera etapa se sembraron 130 peces en cada unidad experimental (6 acuarios) manteniendo las mismas condiciones ambientales y el manejo de la alimentación. Los peces recibieron el alimento con 50 $\mathrm{mg} \mathrm{kg}^{-1}$, un grupo durante dos semanas y el otro durante tres semanas, se aplicaron las mismas tasas de alimentación iniciando con un $20 \%$ de la biomasa por día en 4 raciones con un ajuste semanal, finalizando la última semana con una tasa del $15 \%$.

Evaluación del crecimiento y supervivencia. En ambas etapas la evaluación del crecimiento se realizó con los valores promedios de peso y longitud total y su incremento. La supervivencia fue estimada mediante el conteo total de los peces al final de cada etapa experimental. Se utilizaron las siguientes ecuaciones: 
Longitud total individual promedio $(\mathrm{mm})=\Sigma$ long. individual de peces/número de peces evaluados

Peso individual promedio $(\mathrm{g})=$ Biomasa de cada acuario/número de peces por acuario

Incremento Longitud total promedio $(\mathrm{mm})=\mathrm{Lf}-\mathrm{Li}$

Incremento Peso promedio $(\mathrm{g})=\mathrm{Pf}-\mathrm{Pi}$

Dónde: Li es la longitud total individual promedio inicial, Lf es la longitud total individual promedio final, Pi es el peso total individual promedio inicial, Pf es el peso total individual promedio final.

Supervivencia $S(\%)=\mathrm{N}_{\mathrm{f}} / \mathrm{N}_{\mathrm{i}} \times 100$

Dónde: $\mathrm{N}_{\mathrm{f}}$ es el número de peces al final del experimento, $\mathrm{N}_{\mathrm{i}}$ es el número de peces al inicio del experimento.

Identificación del sexo. Luego del periodo experimental de cada etapa, una muestra de los peces por tratamiento se mantuvo en los acuarios durante 90 días, fueron alimentados con una dieta comercial de 45 $\%$ de proteína, con tasas que variaron desde el 10 al 5 $\%$ diario, para permitir su crecimiento y el desarrollo gonadal para la evaluación del sexo. Luego de este periodo los alevines fueron sacrificados con eugenol disuelto en agua en sobredosis (100 mg/L) y antes de efectuar la necropsia se realizó el corte de la médula, según el método de eutanasia de la CCAC (2010). La necropsia fue realizada de acuerdo con el método descrito por Noga (2010). La identificación del sexo fue realizada mediante la observación al microscopio de una gónada extraída y teñida con aceto-carmín según la metodología de Wassermann y Bertolla (2002) y la segunda gónada fue fijada en formol al $10 \%$ para la preparación de cortes histológicos en la sección Ictiopatología del laboratorio de Histología, Embriología y Patología Veterinaria de la Facultad de Medicina Veterinaria de la Universidad Nacional Mayor de San Marcos del departamento de Lima. Los cortes fueron realizados para la descripción histológica de las gónadas. Para estas evaluaciones fue utilizado un microscopio óptico Eclipse E200 (Nikon Corporation, USA) y las fotografías mediante una cámara digital Coolpix P310 (Nikon Corporation, USA) adaptada al microscopio.
Diseño experimental. Se aplicó para ambos experimentos un diseño completamente al azar (DCA), el primero con 3 tratamientos: Tratamiento 1 (T1): alimentación con una dosis de $50 \mathrm{mg}$ de letrozol por $\mathrm{kg}$ de alimento. Tratamiento 2 (T2): alimentación con una dosis de $100 \mathrm{mg}$ de letrozol por $\mathrm{kg}$ de alimento. Tratamiento 3 (T3): alimentación con una dosis de 200 $\mathrm{mg}$ de letrozol por $\mathrm{kg}^{1}$ de alimento. Un tratamiento Control positivo (C): alimentación con dosis de $60 \mathrm{mg}$ de 17 alfa metil testosterona por $\mathrm{kg}$ de alimento. Un Control negativo ( $\mathrm{C} 0)$ : Alimentación sólo con alimento comercial. La segunda etapa los peces recibieron alimento con una dosis de $50 \mathrm{mg} \mathrm{kg}^{-1}$ de letrozol durante dos periodos de tratamiento $2 \mathrm{~S}$ (dos semanas) y $3 \mathrm{~S}$ (tres semanas). Todos los tratamientos y controles contaron con tres repeticiones.

Análisis estadístico. Las diferencias en los valores promedio de incremento en peso y longitud entre tratamientos y controles fueron evaluados mediante un ANOVA de una sola vía y una comparación con el test de Tuckey. La supervivencia fue comparada por la prueba no paramétrica de Kruskal-Wallis. La eficiencia de masculinización entre tratamientos y controles fue realizada mediante comparación de proporciones macho: hembra con un nivel de significancia de $0.05 \mathrm{Se}$ utilizó el programa Minitab versión 16 y Excel para el procesamiento de los datos.

\section{RESULTADOS Y DISCUSIÓN}

\section{Evaluación del crecimiento y supervivencia entre tratamientos y controles}

La tabla 1 presenta los resultados obtenidos en la evaluación del crecimiento en longitud promedio total, peso total promedio, incremento en longitud e incremento en peso, y supervivencia durante el experimento de evaluación de las tres dosis de letrozol para la masculinización de alevinos de tilapia luego de 4 semanas de experimentación.

Los incrementos en longitud y en peso no mostraron diferencias significativas entre los tratamientos (T1, T2 y T3) ni con los controles positivo y negativo ( $\mathrm{C}$ y $\mathrm{C} 0$ ). Los peces del $\mathrm{C}$ y $\mathrm{T} 1$ presentaron longitudes promedio similares $(27,7 \mathrm{~mm})$ y ligeramente mayores a la de los peces del C0, T2 y T3 $(27,26$ y 26,3 mm). Los pesos promedio al final del experimento fueron 0,$348 ; 0,344$; 
0,$313 ; 0,263$ y $0,284 \mathrm{~g}$ en el T1, C0, C, T2 y T3

respectivamente, con pesos ligeramente mayores en $\mathrm{T} 1$

y control CO.

Tabla 1. Evaluación del crecimiento y supervivencia de alevines de tilapia nilótica ( $O$. niloticus) sometidos a diferentes dosis de letrozol y 17 alfa metiltestosterona durante el periodo de inversión sexual en condiciones de laboratorio.

\begin{tabular}{llllll}
\hline \multirow{2}{*}{ Parámetros } & Controles & \multicolumn{3}{c}{ Tratamientos } \\
\cline { 2 - 6 } & C0 & C & T1 & T2 & T3 \\
\hline Supervivencia (\%)* & $57^{\mathrm{a}}$ & $50^{\mathrm{a}}$ & $62^{\mathrm{a}}$ & $63^{\mathrm{a}}$ & $48^{\mathrm{a}}$ \\
Longitud inicial prom. (mm) & 9,2 & 9,2 & 9,2 & 9,2 & 9,2 \\
Peso inicial prom. (g) & 0,0075 & 0,0075 & 0,0075 & 0,0075 & 0,0075 \\
Longitud final prom.(mm) & 27,0 & 27,7 & 27,7 & 26,0 & 26,3 \\
Peso final prom. (g) & 0,344 & 0,313 & 0,348 & 0,263 & 0,284 \\
Incremento long prom. (mm)* & $17,8^{\mathrm{b}}$ & $18,5^{\mathrm{b}}$ & $18,5^{\mathrm{b}}$ & $16,8^{\mathrm{b}}$ & $17,1^{\mathrm{b}}$ \\
Incremento peso prom. $(\mathrm{g})^{*}$ & $0,336^{\mathrm{c}}$ & $0,306^{\mathrm{c}}$ & $0,341^{\mathrm{c}}$ & $0,255^{\mathrm{c}}$ & $0,277^{\mathrm{c}}$ \\
\hline
\end{tabular}

*nivel de significancia del $5 \%$.

El crecimiento observado bajo las condiciones de temperatura, densidad y tasa de alimentación al ser comparados con otros estudios de condiciones similares está dentro del rango reportado por diversos autores durante la etapa de inversión sexual de $O$. niloticus (Abd Al-Hakim et al., 2013; Drummond et al. 2009; Neumann et al., 2009; Popma y Green, 1990), sin embargo, son menores a los resultados obtenidos por otros autores que trabajaron con diferentes temperaturas, densidades, tasas y frecuencias de alimentación, condiciones ambientales, tratamiento hormonal y variedades de tilapia del Nilo (ChávezGarcía et al., 2020; Rebouças et al., 2014; Rima et al., 2017; Trejo-Quezada et al., 2021). El menor crecimiento obtenido está principalmente relacionado a la densidad utilizada de 3 peces/L en comparación a los valores con densidades de 0,5 a 1 pez/L de los trabajos anteriormente mencionados, Tachibana et al. (2008), al evaluar el crecimiento con densidades de 1 , 3,5 y 7 peces/L, concluyó que los peces alcanzaron un peso final menor conforme la densidad fue mayor.

Los incrementos en peso y longitud entre controles y tratamiento no mostraron diferencias significativas $(\mathrm{p}<0.05)$, el letrozol no presentó un efecto sobre el crecimiento diferente entre las tres dosis estudiadas, ni con la MT y el control C0, resultados similares reportó Betancur et al. (2014) al evaluar la masculinización de tilapia roja con letrozol y exemestano, el crecimiento de los peces no fue significativamente diferente entre los tratamientos y controles, sin embargo, si entre las dosis de los inhibidores, Alanís González, et al. (2014), en los ensayos de validación de inhibidores de aromatasa para la masculinización de tilapia del Nilo, tampoco observó diferencias en el crecimiento de los peces sometidos a tratamientos con exemestano, letrozol, MT y control negativo. Al comparar los resultados con los obtenidos en trabajos realizados para masculinizar tilapia del Nilo con diferentes concentraciones de MT, se encontró que el crecimiento fue mayor conforme se incrementó la dosis o el tiempo de administración de la hormona (Ferdous y Alí, 2011; Trejo-Quezada et al., 2021), tendencia que no se ha encontrado para el letrozol durante la etapa de inversión sexual en el presente trabajo.

El porcentaje de supervivencia promedio y desviación estándar obtenidos al final del experimento fueron 57 $\pm 7,6 ; 50 \pm 13,9 ; 62 \pm 6,8 ; 63 \pm 8,3$ y $48 \pm 11,6$ en $\mathrm{C} 0$, $\mathrm{C}, \mathrm{T} 1, \mathrm{~T} 2$ y $\mathrm{T} 3$ respectivamente sin diferencias significativas $(\mathrm{p}<0.05)$, numéricamente se observó la menor supervivencia en el tratamiento T3 (48\%) y la mayor en T2 (63\%), estos valores son menores a los obtenidos en diversos trabajos de inversión sexual de O. niloticus mencionados anteriormente, no se puede relacionar la mortalidad al efecto del letrozol porque fue similar en los controles y las diferentes dosis del inhibidor. La tabla 2 muestra la mortalidad semanal por controles y tratamientos, notándose un incremento a partir de la segunda semana, al analizar las posibles causas de la mortalidad, se descarta la densidad elegida (3 peces/L) debido a que ésta se mantuvo en el segundo 
experimento y se tuvo una mayor supervivencia al término de las 4 semanas en todos los tratamientos evaluados $(89 \%)$, la diferencia fue el manejo durante las biometrías, los peces muy pequeños fueron afectados por la manipulación durante el pesaje de las muestras en la primera etapa que no fue realizada en la segunda para eliminar el estrés.

\section{Parámetros de calidad del agua}

Los resultados del control de la calidad del agua están presentados en la tabla 3, indicando los valores promedio de la temperatura, el oxígeno disuelto y el $\mathrm{pH}$ por tratamientos y controles.

La temperatura, el oxígeno disuelto y el $\mathrm{pH}$ promedios tuvieron un comportamiento similar en todas las unidades experimentales, las desviaciones típicas observadas entre los tratamientos y controles son cercanas indicando que el manejo de la calidad del agua para mantener estas variables fue adecuado.

Los valores promedio de la temperatura durante las mañanas (cercanos a $28^{\circ} \mathrm{C}$ ) fueron similares a los valores en las tardes $\left(28,2^{\circ} \mathrm{C}\right)$ en los tratamientos y controles con desviaciones típicas entre 1,15 y 0,78 . Drummont et al. (2009), evaluando el efecto de la temperatura en la etapa de inversión sexual de alevinos de tilapia $O$. niloticus, encontraron que ésta no es afectada en un rango entre los 26 y $32^{\circ} \mathrm{C}$, sin embargo, el crecimiento y la supervivencia fueron mejores con temperaturas entre 28,5 y $30^{\circ} \mathrm{C}$. Phelps (2006) menciona que la temperatura óptima para el mantenimiento de los alevines de tilapia durante el periodo de inversión sexual está entre $\operatorname{los} 26$ y $28^{\circ} \mathrm{C}$ y que con temperaturas menores a $24^{\circ} \mathrm{C}$ los peces reducen significativamente el crecimiento y algunos no completan la diferenciación gonadal durante el tratamiento, en el caso opuesto los valores de temperatura mayores a $34^{\circ} \mathrm{C}$ pueden alterar la proporción de sexos en algunas progenies de tilapia incrementándose el número de machos respecto al de las hembras pero con bajas tasas de supervivencia (Azaza, et al., 2013; Baroiller et al., 2009; Soltan, et al., 2013). La temperatura establecida y controlada en esta investigación se mantuvo dentro de los rangos considerados óptimos para el tratamiento hormonal de los peces en todos los tratamientos y controles, no existiendo evidencia de haber sido una variable que pudiese haber afectado el proceso de inversión sexual.

Valores bajos de oxígeno disuelto pueden afectar el proceso de inversión sexual ya que disminuyen el consumo del alimento, la supervivencia y el crecimiento de los peces, los valores de la tabla 2 muestran que la concentración promedio durante las mañanas y las tardes estuvo cercana a 7,0 mg L-1 notándose una leve disminución en la tarde en todos los acuarios; se registró un valor máximo y mínimo de 7,89 y 6,02 $\mathrm{mg} \mathrm{L}^{-1}$ durante un día en los controles C0 y $\mathrm{C}$ respectivamente. Esta relativa diferencia estuvo relacionada a la variación de temperatura que se dio en las unidades experimentales, asimismo no se observó una disminución con el incremento de la biomasa desde la primera semana hasta el final del experimento, confirmando que el sistema de aireación y los recambios de agua permitieron mantener su concentración con muy poca variación. Phelps (2006) recomienda concentraciones mayores a $4 \mathrm{mg} \mathrm{L}^{-1}$ de oxígeno para la inversión del sexo en tilapias, para no afectar el consumo de alimento, así como también para evitar el estrés de los peces y la vulnerabilidad ante las enfermedades. Los valores de oxígeno en el agua se mantuvieron por encima de $6 \mathrm{mg} \mathrm{L}^{-1}$ por tal razón se considera que no hubo un efecto negativo en el consumo del alimento o bienestar de los peces.

Tabla 2. Variación promedio y desviación estándar por semanas del número de peces debido a la mortalidad en los tratamientos y controles $(n=3)$.

\begin{tabular}{lccccc}
\hline & \multicolumn{3}{c}{ Controles } & Tratamientos \\
\cline { 2 - 6 } $\mathbf{N}^{\circ}$ peces & $\mathbf{C 0}$ & $\mathbf{C}$ & $\mathbf{T 1}$ & $\mathbf{T 2}$ & $\mathbf{T 3}$ \\
\hline Inicial & 130 & 130 & 130 & 130 & 130 \\
$1^{\mathrm{a}}$ semana & $127 \pm 0.57$ & $129 \pm 1$ & $128 \pm 1$ & $128 \pm 1$ & $128 \pm 1.5$ \\
$2^{\mathrm{a}}$ semana & $108 \pm 9$ & $111 \pm 8$ & $99 \pm 8$ & $114 \pm 5$ & $105 \pm 8$ \\
$3^{\mathrm{a}}$ semana & $86 \pm 10$ & $77 \pm 17$ & $83 \pm 8$ & $93 \pm 9$ & $76 \pm 10$ \\
$4^{\mathrm{a}}$ semana & $74 \pm 10$ & $65 \pm 18$ & $80 \pm 9$ & $82 \pm 11$ & $62 \pm 15$ \\
\hline
\end{tabular}

C0: control negativo, C: MT $60 \mathrm{mg} \mathrm{kg}^{-1}$, T1: letrozol $50 \mathrm{mg} \mathrm{kg}^{-1}$, T2: letrozol $100 \mathrm{mg} \mathrm{kg}^{-1}$, T3: letrozol $200 \mathrm{mg} \mathrm{kg}$ 1 
Vega, E., Sandoval, N., \& Angeles, B. (2021). Anales Científicos. 82(2), 262-278. DOI. 10.21704/ac.v82i2.1789

Tabla 3. Valores promedio y desviación estándar $(n=3)$ de la temperatura, el oxígeno disuelto (mañana y tarde) y el $\mathrm{pH}$ (tarde) por tratamientos y controles durante la fase experimental de la inversión sexual de $O$. niloticus con letrozol a diferentes concentraciones.

\begin{tabular}{llllll}
\hline & \multicolumn{2}{c}{ Controles } & \multicolumn{2}{c}{ Tratamientos } \\
\cline { 2 - 6 } Parámetros & $\mathbf{C}$ & C0 & T1 & T2 & T3 \\
\hline Temperatura mañana $\left({ }^{\circ} \mathrm{C}\right)$ & $28,16 \pm 1,15$ & $28,03 \pm 0,84$ & $28,04 \pm 0,89$ & $28,09 \pm 0,83$ & $28,05 \pm 1,12$ \\
Temperatura tarde $\left({ }^{\circ} \mathrm{C}\right)$ & $28,28 \pm 0,99$ & $28,1 \pm 0,78$ & $28,22 \pm 0,89$ & $28,21 \pm 0,83$ & $28,22 \pm 0,96$ \\
Oxígeno mañana $\left(\mathrm{mg} . ~^{-1}\right)$ & $7,10 \pm 0,29$ & $7,07 \pm 0,26$ & $7,06 \pm 0,22$ & $7,09 \pm 0,25$ & $7,11 \pm 0,26$ \\
Oxígeno tarde $\left(\mathrm{mg} . \mathrm{L}^{-1}\right)$ & $6,99 \pm 0,34$ & $6,95 \pm 0,31$ & $6,97 \pm 0,26$ & $6,98 \pm 0,29$ & $7,05 \pm 0,24$ \\
pH & $7,95 \pm 0,16$ & $7,93 \pm 0,10$ & $7,95 \pm 0,10$ & $7,95 \pm 0,10$ & $7,97 \pm 0,08$ \\
\hline
\end{tabular}

\section{Inversión sexual}

Los resultados de la proporción de machos y hembras por tratamientos y controles son mostrados en la tabla 4 , donde se indica el número de peces evaluados y los porcentajes de cada sexo obtenidos.

Los porcentajes de machos con las tres dosis de letrozol fueron diferentes significativamente $(\mathrm{p}<0.05)$ a los obtenidos con MT y con el control negativo, la proporción de machos con MT fue mayor al C0.

Kwon et al. (2000), al utilizar el fadrozole con dosis mayores a $200 \mathrm{mg} \mathrm{kg}^{-1}$ de alimento reportó resultados de masculinización mayores al 96 por ciento para la tilapia del Nilo, Afonso et al. (2001) al emplear concentraciones de fadrozole de 75 a $100 \mathrm{mg} \mathrm{kg}^{-1}$ logró una masculinización del 100 por ciento en alevinos de O. niloticus. Das et al. (2012) en ensayos con letrozol consiguió el 97 y 100 por ciento de alevinos machos de tilapia mosambica (O. mossambicus) con niveles de 100 y $200 \mathrm{mg} \mathrm{kg}^{-1}$ y al usar dos combinaciones de letrozol y MT (50 mg kg-1 $+25 \mathrm{mg} \mathrm{kg}^{-1}$ y $100 \mathrm{mg} \mathrm{kg}^{-1}$ $+25 \mathrm{mg} \mathrm{kg}^{-1}$ ) 92,3 y 100 por ciento correspondientemente. Betancur et al. (2014), en experimentos de masculinización con alevinos de tilapia roja (Oreochromis spp.) con exemestano y letrozol, dos inhibidores de aromatasa de tercera generación de tipo I y II, reportó 92,6 y menos de 80 $\%$ con dosis de letrozol de 100 y $25 \mathrm{mg} \mathrm{kg}^{-1}$. Trabajos recientes con el uso de letrozol en otras especies

Tabla 4. Número y porcentaje promedio de peces machos, hembras e intersexo de alevines de O. niloticus luego del tratamiento con diferentes dosis de letrozol (50, 100 y $\left.200 \mathrm{mg} \mathrm{kg}^{-1}\right)$ y controles.

\begin{tabular}{llllllll}
\hline $\begin{array}{l}\text { Tratamientos } \\
\text { y controles }\end{array}$ & $\begin{array}{l}\mathbf{N}^{\mathbf{0}} \\
\text { peces }\end{array}$ & $\begin{array}{l}\mathbf{N}^{\mathbf{0}} \\
\text { machos }\end{array}$ & $\begin{array}{l}\mathbf{N}^{\mathbf{o}} \\
\text { hembras }\end{array}$ & $\begin{array}{l}\mathbf{N}^{\mathbf{0}} \\
\text { intersexo }\end{array}$ & $\begin{array}{l}\% \\
\text { machos }\end{array}$ & $\begin{array}{l}\% \\
\text { hembras }\end{array}$ & $\begin{array}{l}\% \\
\text { intersexo }\end{array}$ \\
\hline & & & & & & & \\
$\mathrm{T} 1$ & 33 & 30 & 1 & 2 & $90^{\mathrm{a}}$ & 3 & 7 \\
$\mathrm{~T} 2$ & 32 & 32 & 0 & 0 & $100^{\mathrm{a}}$ & 0 & 0 \\
$\mathrm{~T} 3$ & 24 & 23 & 0 & 1 & $93^{\mathrm{a}}$ & 0 & 7 \\
$\mathrm{C}$ & 28 & 19 & 9 & 0 & $66^{\mathrm{b}}$ & 29 & 5 \\
$\mathrm{C} 0$ & 36 & 16 & 20 & 0 & $45^{\mathrm{c}}$ & 55 & 0 \\
\hline
\end{tabular}

Nivel de significancia 0.05, n=3. C0: control negativo, C: MT 60 mg kg-1, T1: letrozol 50 mg kg-1, T2: letrozol 100 mg kg-1, T3: letrozol 200 mg kg-1

Los resultados de masculinización obtenidos en este trabajo con el letrozol en dosis de 50, 100 y 200 mg.kg1 de alimento administrado durante 4 semanas y los obtenidos en los trabajos anteriormente mencionados muestran que la eficiencia de masculinización del letrozol vía oral es dependiente de las dosis administradas. Este efecto en función de la dosis también fue logrado con el fadrozole durante la masculinización de alevinos de $\mathrm{O}$. niloticus reportado por Kwon et al. (2000) quienes evaluaron tratamientos con niveles entre 40 a $500 \mathrm{mg} \mathrm{kg}-1$ de alimento, encontrando que las dosis a partir de $200 \mathrm{mg} \mathrm{kg-1} \mathrm{de}$ alimento permitió la masculinización de más del 90 por ciento de los peces, es notable la mayor potencia del letrozol respecto al fadrozole, el cual con menores concentraciones en el alimento logra un mayor número 
de peces machos. Por este mejor efecto con dosis reducidas del letrozol es que se le considera un inhibidor de aromatasa de tercera generación (Bhatnagar, 2007).

$\mathrm{Xu}$ et al. (2021) al investigar sobre los mecanismos moleculares de la masculinización gonadal de poblaciones hembras triploides $(\mathrm{XXX})$ de trucha arcoiris (Oncorhynchus mykiss) presentó resultados luego de administrar a los peces dosis de $1.5 \mathrm{mg} \mathrm{kg}^{-1} \mathrm{de}$ MT y $50 \mathrm{mg} \mathrm{kg}^{-1}$ de letrozol durante 377 días después de la fertilización (dpf), en los primeros días del tratamiento (56 a $80 \mathrm{dpf}$ ), no se observó efecto sobre el desarrollo del ovario, sin embargo, el letrozol fue más eficiente en la masculinización de la gónada después de los 94 hasta los 377 dpf, al finalizar el experimento se encontró que la expresión de los genes asociados a la conformación de testículos (sdy, mrt1, sox9 y amh) tuvo un aumento significativo en el grupo que fue tratado con letrozol en comparación a los peces que recibieron MT y al control, los niveles fueron similares al de los peces machos diploides, la expresión de los genes asociados a la conformación de ovarios (cyp19a1a y fox12) fue inhibida significativamente a los $377 \mathrm{dpf}$, los autores concluyeron que el letrozol indujo eficientemente la inversión del sexo de hembras triploides de trucha arcoíris.

Respecto a los resultados con la hormona $17 \mathrm{MT}$, el control positivo en este experimento, el porcentaje promedio de peces machos (66 por ciento) fue diferente significativamente a los valores de las tres dosis de letrozol, este porcentaje es muy bajo en comparación a los obtenidos en la mayoría de trabajos realizados con MT y tilapia del Nilo, donde se obtuvieron resultados mayores al 90 por ciento con dosis administradas entre 50 y $60 \mathrm{mg} \mathrm{kg}^{-1} \mathrm{de}$ (Abd AlHakim et al., 2013; Ferdous y Ali, 2011; Phelps y Popma, 2000; Chávez-García et al., 2020; Rebouças et al., 2014; Rima et al., 2017; Trejo-Quezada et al., 2021). La menor eficiencia promedio alcanzada en este experimento puede ser explicada principalmente por la variación del tamaño de los peces que fue observada (coeficiente de variación de la longitud total de los peces 26\%), a pesar de que teóricamente se ajustó la tasa de alimentación semanalmente para que la cantidad del alimento sea la requerida para el crecimiento y la masculinización de toda la población, sin embargo, la presencia de peces dominantes pudo impedir un consumo individual homogéneo del alimento, por lo que las dosis efectivas de hormona que recibieron los peces denominados subordinados fue menor, la presencia de hembras (29\%) e individuos con gónadas intersexo $(5 \%)$ puede estar relacionado al menor consumo del alimento con hormona. El crecimiento heterogéneo en peces territoriales como O. niloticus es explicado por Azaza et al. (2013), al evaluar el efecto de la densidad sobre el crecimiento, la homogeneidad del tamaño y la variación del consumo de alimento en cultivos de $O$. niloticus, los autores encontraron diferencias en el consumo de alimento entre los peces dominantes y los subordinados, observando mayor consumo, crecimiento más rápido, mayor tamaño en los peces dominantes y en los subordinados observaron estrés, disminución del apetito, mayores costos metabólicos debido a la menor oportunidad de alimentarse. Este experimento ha utilizado una densidad inicial alta 3 peces/L pudiéndose haber disminuido la heterogeneidad (Tachibana et al., 2008) que se presenta en densidades bajas, sin embargo, considerando la mortalidad que se tuvo a partir de la segunda semana, la densidad real en los acuarios disminuyó hasta 1.5 peces/L en la tercera y cuarta semana. Otro aspecto que debe ser considerado está relacionado a la frecuencia de alimentación utilizada de 4 veces al día, diversos autores mencionan que mejores resultados son obtenidos con frecuencias de 6 veces al día y tasas decrecientes a partir de la primera semana (Abu Zeid y Ali, 2015; Rima et al., 2017; Trejo-Quezada et al., 2021), en conclusión la interacción entre el manejo de la alimentación, la manipulación durante los muestreos, el estrés y la mortalidad que se presentó en el primer experimento de forma similar para todos los tratamientos y controles afectó en mayor proporción la inversión sexual con la MT.

Los resultados del control negativo fueron diferentes significativamente al porcentaje obtenido con los peces del control positivo (17 MT) y con las tres dosis de letrozol, estos valores se encuentran dentro de lo esperado al tener una proporción similar entre individuos machos y hembras (55 y $45 \%$ ) o una proporción cercana a 1:1 como es frecuentemente observado en los experimentos sobre inversión sexual con tilapia del Nilo, mencionados anteriormente. 


\section{Descripción de gónadas}

La observación de las gónadas permitió la identificación adecuada del sexo en cada uno de los ejemplares evaluados. La figura 1 ilustra la diferencia entre los tejidos de gónadas masculina, femenina e intersexo en muestras analizadas, en las gonadas masculinas se observaron los lóbulos testiculares con diferentes grados de desarrollo, en las gónadas de las hembras se visualizaron oocitos y en gónadas intersexo la presencia de oocitos distribuidas entre el tejido testicular.

Al analizar las muestras histológicas de gónadas masculinas de peces sometidos a los tratamientos y controles se pudo observar predominantemente peces en estadio 2 o en desarrollo, de acuerdo a las clases o estadios sugeridos y adaptados por diversos autores (Núñez y Duponchelle 2009; Brown-Peterson et al. 2011 y Kosai et al. 2011). Las gónadas en estadio 2 están caracterizadas por poseer numerosos cistos bien organizados con diferentes estadios espermatogénicos que van desde espermatogonias hasta espermátides, como se muestra en la Figura 2.

Dentro de cada tratamiento y controles los peces presentaron diferentes estados de maduración pudiéndose identificar peces del estadio 1 al 3. Las gónadas en estadio 1 son inmaduras y se reconocen por la ausencia de la actividad espermatogénica en el tejido intersticial y la presencia de espermatocitos primarios y el estadio 3 con una espermatogénesis intermedia y presencia de espermatocitos, espermátides y espermatozoos en proporciones similares. No se puede relacionar estas diferencias con algún efecto de los tratamientos, debido a que no se determinó el número de peces de cada estadio, la presencia de algunos peces en un estado más avanzado de madurez gonadal se puede relacionar al crecimiento heterogéneo de los peces, los peces de mayor tamaño por dominancia del grupo pueden madurar primero. Se conoce la influencia de las gónadas en el crecimiento de los peces teleósteos como es el caso de las tilapias del género Oreochromis (Bhatta et al. 2012).

\section{Evaluación del tiempo de aplicación del} tratamiento con letrozol

Los resultados de la inversión sexual aplicando diferentes tiempos de tratamiento con letrozol a una dosis de $50 \mathrm{mg} \mathrm{kg}^{-1}$ de alimento son presentados en la tabla 5 , se puede apreciar que los porcentajes de masculinización fueron 73 y $82 \%$ en los tratamientos de dos y tres semanas respectivamente. Estos valores son menores a los obtenidos en el primer experimento de cuatro semanas donde con esta dosis se alcanzó el $90 \%$ de masculinización (Figura 3).

Afonso et al. (2001) en ensayos con el fadrozole para la masculinización de $O$. niloticus, con dosis de 50, 75 y $100 \mathrm{mg} \cdot \mathrm{kg}^{-1}$ de alimento, obtuvo resultados del 67, 76 y $90 \%$ respectivamente, después de 15 días de tratamiento y porcentajes de 77, 100 y 100 a los 30 días de alimentación con esas mismas dosis. Comparando con los resultados del presente trabajo se aprecia que con $50 \mathrm{mg}$ de letrozol por $\mathrm{kg}$ de alimento administrado durante 15 días de tratamiento el porcentaje de machos fue mayor al obtenido con la misma dosis de fadrozole (73 vs. 67 por ciento) así como también a los 30 días (90 vs. 77 por ciento), observándose una mayor potencialidad de inhibición del letrozol.

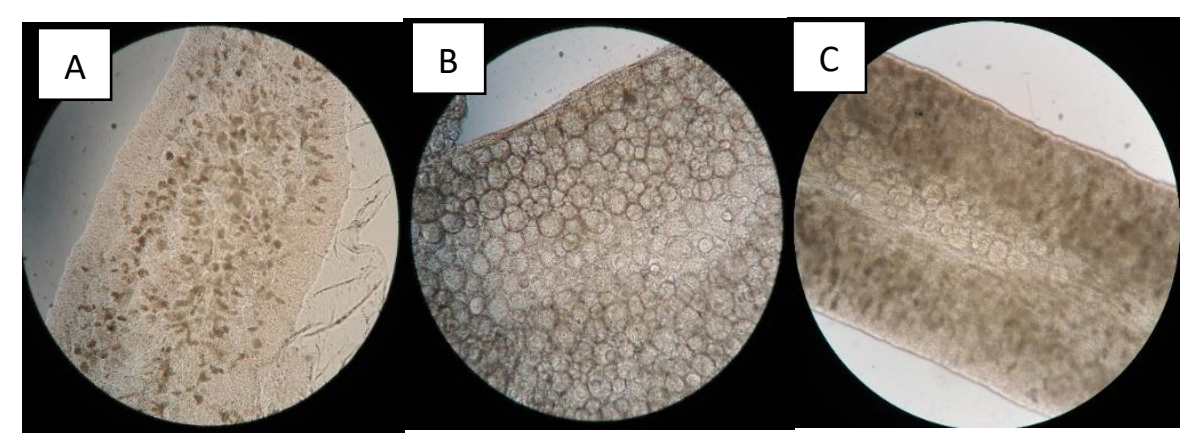

Figura 1. Observación microscópica en fresco de las gónadas de tilapia de individuos machos (A), hembras (B) e intersexo (C) 100X. 
Vega, E., Sandoval, N., \& Angeles, B. (2021). Anales Científicos. 82(2), 262-278. DOI. 10.21704/ac.v82i2.1789

J

\section{Estadio 1}

$\mathrm{C} 0$
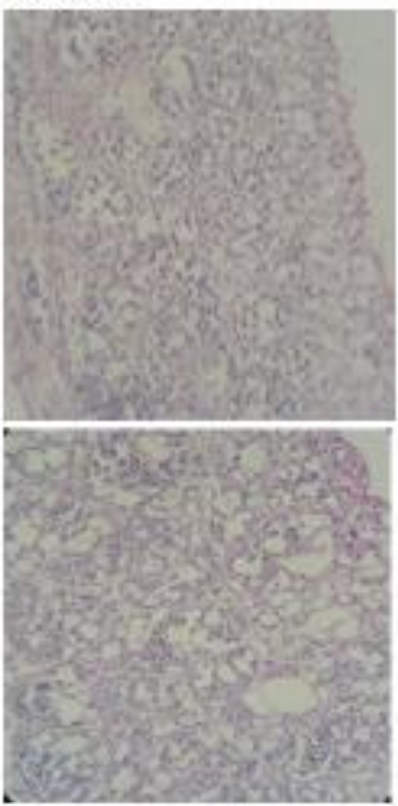

C

T1
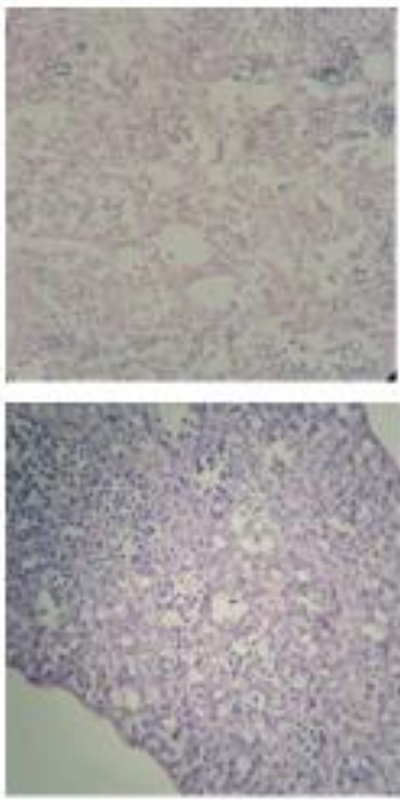

$\mathrm{T} 2$

T3

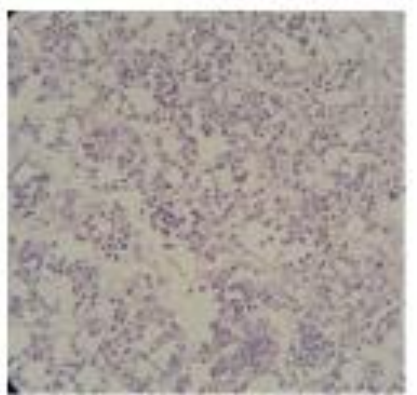

\section{Estadio 2}
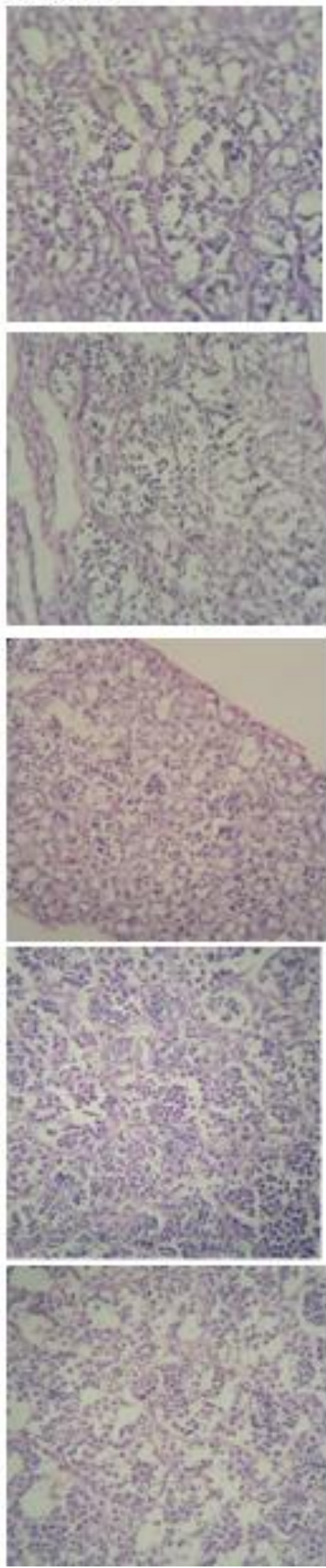

\section{Estadio 3}
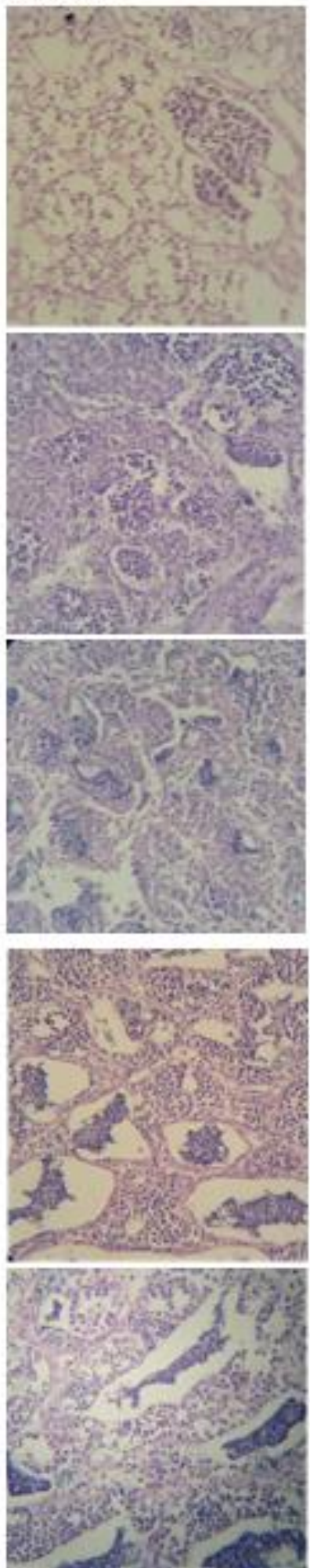

Figura 2. Cortes histológicos de gónadas masculinas de alevinos de $O$. nitoticus con diferentes estados de desarrollo por tratamientos con letrozol T1, T2, T3 (50, 100 y $\left.200 \mathrm{mg} \cdot \mathrm{kg}^{-1}\right), \mathrm{C}$ (60 mg. $\mathrm{kg}^{-1} \mathrm{de} \mathrm{MT)} \mathrm{y} \mathrm{C0} \mathrm{(solo} \mathrm{alimento).} \mathrm{400X.}$ 
Vega, E., Sandoval, N., \& Angeles, B. (2021). Anales Científicos. 82(2), 262-278. DOI. 10.21704/ac.v82i2.1789

Tabla 5. Resultados de la evaluación del sexo de alevines de O. niloticus sometidos a tratamiento con $50 \mathrm{mg}$ de letrozol por $\mathrm{kg}$ de alimento durante dos $(2 \mathrm{~S})$ y tres semanas $(3 \mathrm{~S})$.

\begin{tabular}{llllll}
\hline Tratamiento & $\mathrm{N}^{\circ}$ peces & $\mathrm{N}^{\circ}$ machos & $\mathrm{N}^{\circ}$ hembras & $\%$ machos & $\%$ hembras \\
\hline $2 \mathrm{~S}$ & 115 & 84 & 31 & $73 \pm 3,7^{\mathrm{a}}$ & 27 \\
$3 \mathrm{~S}$ & 114 & 94 & 20 & $82 \pm 10,58^{\mathrm{b}}$ & 18 \\
\hline
\end{tabular}

Nivel de significancia 0.05 .

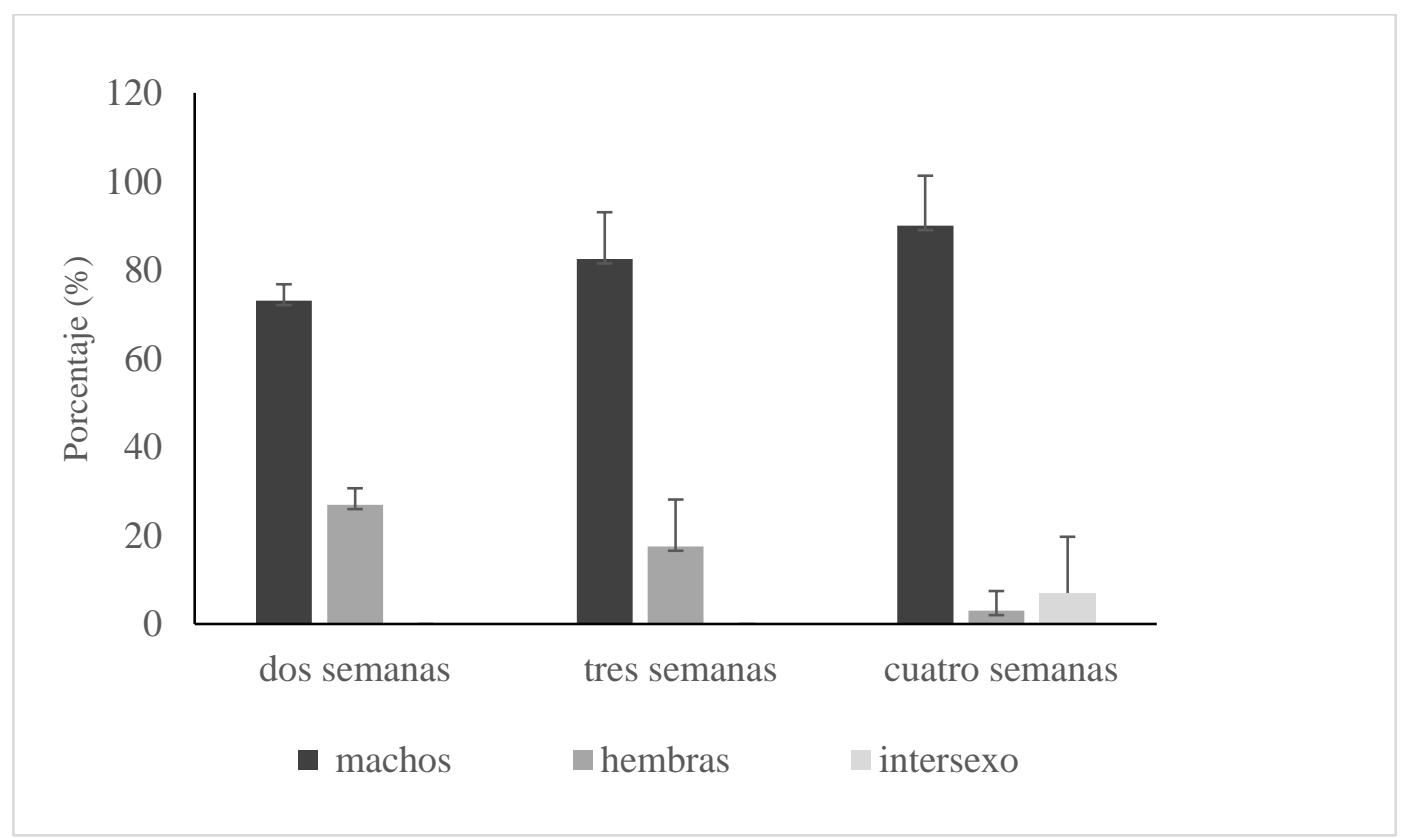

Figura 3: Porcentaje promedio y desviación estándar de machos, hembras e intersexo obtenidos luego del tratamiento con letrozol en el alimento $\left(50 \mathrm{mg} \mathrm{kg}^{-1}\right)$ durante dos, tres y cuatro semanas de tratamiento $(\mathrm{n}=3)$.

El incremento de peces machos con relación al tiempo de administración puede ser explicado por el mecanismo de acción del letrozol, un inhibidor enzimático no esteroideo que se une reversiblemente al sitio activo de la enzima y actúa mientras la concentración del inhibidor sea suficiente para tener ocupado el sitio de unión enzimática. Cuando es utilizado en pacientes humanos se reporta que la vida media de eliminación es de 42 horas y su concentración estable puede ser alcanzada entre dos a seis semanas, lo que hace que su efecto sea dependiente de la concentración y del tiempo de administración (Bhatnagar 2007).

Afonso et al. (2000) reportaron el efecto del fadrozole en la disminución de la concentración del E2 y el incremento de andrógenos y progestágenos en el plasma de los salmones, Singh y Srivastava (2014) luego de aplicar letrozol en dosis de 100 y $200 \mathrm{mg} \mathrm{kg}$ ${ }^{1}$ durante 60 días a alevinos de tilapia (O. niloticus) encontraron concentraciones disminuidas de estradiol (E2) en plasma comparados con los peces de control, observando un declive notable en la relación E2/Testosterona de 0,266 a 0,076 con la mayor dosis y concentraciones mínimas de E2 (85,83 pg.ml-1) aun después del tratamiento. Estos resultados pueden explicar que con dosis menores administradas durante periodos cortos no se logra la masculinización del 100 $\%$ de la población.

Los primeros signos de la diferenciación sexual en $O$. niloticus ocurren entre los 20 y 25 días después de la eclosión (Kobayashi et al., 2013; Piferrer, 2001) y considerando que éste experimento se inició con peces de 6 días después de la eclosión, al suministrar dosis de $50 \mathrm{mg} \mathrm{kg}^{-1}$ durante dos semanas (2S) se encontró un 
Vega, E., Sandoval, N., \& Angeles, B. (2021). Anales Científicos. 82(2), 262-278. DOI. 10.21704/ac.v82i2.1789

mayor porcentaje de hembras $(27 \%)$ que a las tres semanas $(18 \%)$ y a las 4 semanas de tratamiento $(3 \%)$.

Debido al mecanismo de acción del letrozol en función a dosis y tiempo de aplicación y considerando que para O. niloticus la meiosis ovárica se desarrolla entre los 25 y 30 días poseclosión (Kobayashi et al., 2013) y la plasticidad de las gónadas para invertir su sexo es hasta después de haber completado la diferenciación sexual (Paul-Prasanth et al., 2013; Sun et al., 2014), los tratamientos con dosis de $50 \mathrm{mg} \mathrm{kg}^{-1}$ pueden ser mayores a 4 semanas para una mayor eficiencia de la masculinización. En relación a lo mencionado, al comparar los resultados con la hormona 17 alfa metiltestosterona $\mathrm{u}$ otros andrógenos aplicados en dosis mayores a $60 \mathrm{mg} \mathrm{kg}^{-1}$ y por tiempos mayores a las 4 semanas (Phelps y Okoko, 2011; Piferrer, 2001), para el letrozol no se han reportados casos de la mal denominada "feminización paradójica" donde se aprecia que dosis elevadas de andrógenos disminuyen la proporción de machos obtenidos en el tratamiento.

Para concluir que el letrozol puede ser utilizado como un método alternativo al uso de andrógenos para la masculinización de $O$. niloticus, es necesario continuar con estudios para definir dosis y tiempos mínimos de efectividad considerando los costos altos del producto en comparación a MT y estudios del impacto hacia el medio ambiente como su persistencia en sedimentos y agua.

\section{CONCLUSIONES}

El uso del inhibidor de aromatasa letrozol en el tratamiento oral para la inversión del sexo de alevines de $O$. niloticus en el rango de 50 a $200 \mathrm{mg} \mathrm{kg}^{-1}$ de alimento no afectó el crecimiento ni la supervivencia y fue efectivo en la obtención de más de $90 \%$ de machos bajo las condiciones experimentales de esta investigación. El tratamiento con la menor dosis de 50 $\mathrm{mg} \mathrm{kg}{ }^{-1}$ requiere como mínimo 4 semanas para la masculinización de $90 \%$ de la población.

\section{Agradecimiento}

Los autores agradecen al Consejo Nacional de Ciencia, Tecnología e Innovación Tecnológica por el apoyo económico para la ejecución al proyecto especial 3982012.

\section{Conflictos de intereses}

Los autores firmantes del presente trabajo de investigación declaran no tener ningún potencial conflicto de interés personal o económico con otras personas $\mathrm{u}$ organizaciones que puedan influir indebidamente con el presente manuscrito.

\section{Contribuciones de los autores}

Preparación y ejecución: EV, BA; Desarrollo de la metodología: EV, NS, BA; Concepción y diseño: EV, NS, BA; Edición del artículo: EV, BA; Supervisión del estudio: EV, BA.

\section{REFERENCIAS}

- Akaike, H. (1974). A new look at the statistical Abd Al-Hakim, N., Hessen, M., Rizkalla, E., Hegazi, A., Tahoun, A., \& Khalfalla, A. (2013). Comparative study for the production of the male Nile tilapia between inter-specific hybridization and hormonal sex reversal. Egyptian Journal of Aquatic Biology and Fisheries, 17(2), 73-89. https://doi.org/10.12816/0011034.

- Abou Zied, R., Ali, A. (2015). Effect of feeding rate and frequency on growth performance, sex conversion ratio and profitability of nile tilapia (Oreochromis niloticus) fry in hapa at commercial hatcheries. Egyptian Journal of Nutrition and Feeds, 18(Issue 2 Special), 451-459. https://doi.org/10.21608/ejnf.2015.104520

- Afonso, L.O.B., Iwama, G.K., Smith, J., \& Donaldson, E.M. (2000). Effects of the aromatase inhibitor Fadrozole on reproductive steroids and spermiation in male coho salmon (Oncorhynchus kisutch) during sexual maturation. Aquaculture 188, 175-187. https://doi.org/10.1016/S00448486(00)00335-5

- Afonso, L.O.B., Wassermann, G.J., \& Terezinha de Oliveira, R. (2001). Sex reversal in Nile tilapia (Oreochromis niloticus) using a nonsteroidal aromatase inhibitor. Journal of Experimental $\begin{array}{llll}\text { Zoology } & 290 & \text { (2), }\end{array}$ 181. https://doi.org/10.1002/jez.1047

- Alanís González, A., Rodríguez Montes de Oca, G. A., Brito Martínez, X. G., \& Román Reyes, J. C. (2014). Validación del uso de inhibidores sintéticos de la aromatasa en la masculinización de la tilapia 
Oreochromis niloticus. Revista Biológico Agropecuaria Tuxpan, 2(1), 434-440. https://doi.org/10.47808/revistabioagro.v2i1.331

- Azaza, M., Assad, A., Maghrbi, W., \& El-Cafsi, M. (2013). The effects of rearing density on growth, size heterogeneity and inter-individual variation of feed intake in monosex male Nile tilapia Oreochromis niloticus L. Animal, 7(11),18651874. https://doi.org/10.1017/S1751731113001493

- Baltazar, P., Mendoza, D. \& Castañeda, M. (2018). Tilapia Potential in Peru. Word Aquaculture Magazine. $\quad$ 49(3):35-40. https://www.was.org/Magazine/Vol/49/3\#.YcrLD GjMJPY

- Barry, T., Marwah, A., \& Marwah, P. (2007). Stability of $17 \alpha$-methyltestosterone in fish feed. Aquaculture 271,1- 4. https://doi.org/10.1016/j.aquaculture.2007.05.001

- Baroiller, J.F., D'Cotta, H., Bezault, E., Wessels, S., \& Hoerstgen-Schwark, G. (2009). Tilapia sex determination: Where temperature and genetics meet. Comparative Biochemistry and Physiology, Part A: Molecular \& Integrative Physiology 153 (1), 30-38. https://doi.org/10.1016/j.cbpa.2008.11.018

- Bhatta, S., Iwai, T., Miura, C., Higuchi, M., Shimizu- Yamaguchi, S., Fukuda, H., \& Miura, T. (2012). Gonads directly regulate growth in teleosts. Proceedings of the National Academy of Sciences of the United States of America, www.pnas.org/lookup/suppl/doi:10. 1073/pnas.1118704109

- Bhatnagar, A.S. (2007). The discovery and mechanism of action of letrozole, Breast Cancer Research and Treatment 105(1), 7-17. https://doi.org/10.1007/s10549-007-9857-4

- Beardmore J.A., Mair G.C., \& Lewis R.I. (2001). Monosex male production in finfish as exemplified by tilapia: applications, problems, and prospects. Aquaculture 197, 283-301. https://doi.org/10.1016/S0044-8486(01)00590-7

- Betancur, J.J., Quintero, J.C., Ostos, H., BarreiroSanchez, F., \& Olivera-Angel, M. (2014). Effectiveness of the aromatase (P450 Arom) inhibitors Letrozole and Exemestane for masculinization of red tilapia (Oreochromis spp.).
Revista Colombiana de Ciencias Pecuarias, ISSNe 0120-0690, 27 (1), 47-53.

- Bombardelli R.A., Hayashi C., \& Meurer F. (2004). Aplicacao de metodos diretos e indiretos para a producao de populacoes monossexuais na tilapicultura. Arquivos de Ciências Veterinárias e Zoologia da UNIPAR 7 (1),57-68. https://revistas.unipar.br/index.php/veterinaria/arti cle/view/545

- Brown-Peterson, N. J., Wyanski, D. M., SaboridoRey, F., Macewicz, B. J., \& Lowerre-Barbieri, S.K. (2011). A Standardized Terminology for Describing Reproductive Development in Fishes, Marine and Coastal Fisheries, 3(1), 52-70. https://doi.org./10.1080/19425120.2011.555724

- Canadian Council on Animal Care (2010). CCAC guidelines on: Euthanasia of animals used in science. $32 \quad p$. https://www.ccac.ca/Documents/Standards/Guidel ines/Euthanasia.pdf

- Das R., Rather M.A., Basavaraja, N., Sharma R., \& Udit U.K. (2012). Effect of Nonsteroidal Aromatase Inhibitor on Sex Reversal of Oreochromis mossambicus (Peters, 1852). Israeli Journal of Aquaculture-Bamidgeh, 64(4),1-6. http://hdl.handle.net/10524/22926

- Drummond, C. D., Murgas, L. D. S., \& Vincetini, B. (2009). Growth and survival of tilapia Oreochromis niloticus (Linnaeus, 1758) submitted to different temperatures during the process of sex reversal. Ciência e Agrotecnologia, 33(3), 895902. https://doi.org/10.1590/S141370542009000300033.

- El-Greisy, Z. A., \& El-Gamal, A. E. (2012). Monosex production of tilapia, Oreochromis niloticus using different doses of $17 \alpha$ methyltestosterone with respect to the degree of sex stability after one year of treatment. The Egyptian Journal of Aquatic Research,38(1), 59-66. https://doi.org/10.1016/j.ejar.2012.08.005

- El-Sayed, A., El-Sayeda H., \& Heba AG. (2012). Effects of phytoestrogens on sex reversal of Nile tilapia (Oreochromis niloticus) larvae fed diets treated with $17 \alpha$-Methyltestosterone Aquaculture 360-361. 58-63. https://doi.org/10.1016/j.aquaculture.2012.07.010

- Ferdous, Z., \& Ali, M. M. (2011). Optimization of hormonal dose during masculinization of tilapia 
(Oreochromis niloticus) fry. Journal Bangladesh Agriculture University Journal, 9(2), 359-364. https://doi.org/10.22004/ag.econ.208687

- Gao, Z.X., Wang, H.P, Wallat, G., Yao, H., Rapp, D., O’Bryant, P., MacDonald, R., \& Wang W.M. (2010). Effects of a nonsteroidal aromatase inhibitor on gonadal differentiation of bluegill sunfish Lepomis macrochirus. Aquaculture Research, 41: 1282-1289. https://doi.org/10.1111/j.1365-2109.2009.02414.x

- Green, B.W., Teichert-Coddington, D.R. (2000). Human Food Safety and Environmental Assessment of the Use of $17 \alpha$-Methyltestosterone to Produce Male Tilapia in the United States. Journal of the World Aquaculture Society, 31: 337$357 . \quad$ https://doi.org/10.1111/j.17497345.2000.tb00885.x

- Hiott, A., \& Phelps, R.P. (1993) Effects of initial age and size on sex reversal of Oreochromis niloticus fry using methyltestosterone. Aquaculture 112:4. https://doi.org/10.1016/00448486(93)90391-B

- Katare, M. B., Basavaraja, N., Joshi, H. D., \& Archana, C. (2015). Effect of letrozole on masculinization of Siamese fighting fish (Betta splendens). Journal of Applied and Natural Science, 7(1), 425-433. https://doi.org/10.31018/jans.v7i1.627

- Klipp, S. P., Pereira, M. O., \& Jatobá, A. (2019). Influência da frequência alimentar durante inversão sexual da tilápia do nilo. Revista Científica Rural, $21: 1$, p. 205-216. https://doi.org/10.30945/rcrv21i1.304

- Kobayashi Y., Nagahama Y., \& Nakamura M. (2013). Diversity and Plasticity of Sex Determination and Differentiation in Fishes. Sexual Development 7:115-125. https://doi.org/10.1159/000342009

- Kosai, P., Jiraungkoorskul, W., Sachamahithinant, C., \& Jiraungkoorskul, K. (2011). Induction of testis-ova in nile tilapia (Oreochromis niloticus) exposed to 17 $\beta$-estradiol. Natural Science, 3(03), 227. http://dx.doi.org/10.4236/ns.2011.33029

- Kwon, J.Y., Haghpanah, V., Kogson-Hurtado, L.M., McAndrew, B.J., \& Penman, D.J. (2000). Masculinization of genetic female Nile tilapia (Oreochromis niloticus) by dietary administration of an aromatase inhibitor during sexual differentiation. Journal of Experimental Zoology, 287 (1): 46-53. https://doi.org/10.1002/1097010X(20000615)287:1<46::AID-JEZ6>3.0.CO;2$\mathrm{X}$

- Macintosh D. (2010). Risks Associated with Using Methyl Testosterone in Tilapia Farming. Disponible en http://media.sustainablefish.org/MT_WP.pdf

- Mainardes-Pinto C.S.R., Fenerich-Verani N., Campos B.E.S., \& Silva A.L. (2000). Masculinização da tilápia do Nilo, Oreochromis niloticus, utilizando diferentes rações e diferentes doses de 17 a-metiltestosterona. Revista Brasileira de Zootecnia, 29: 3 pp. 654-659. https://doi.org/10.1590/S151635982000000300003.

- Mlalila, N., Mahika, C.G., Kalombo, L., Swai, H.S., \& Hilonga, A. (2015). Human food safety and environmental hazards associated with the use of methyltestosterone and other steroids in production of all-male tilapia. Environmental Science and Pollution Research, 22, 4922-4931. https://doi.org/10.1007/s11356-015-4133-3

- Meurer, F., Bombardelli R.A., Santana da Paixao P., Rosa da Silva, L.C., \& Dena dos Santos, L. (2012). Feeding frequency on growth and male percentage during sexual reversion phase of Nile tilapia. Revista Brasileira de Saúde e Produção Animal 13: 4, pp. 1133-1142. http://www.rbspa.ufba.br

- Neumann, E., Ribeiro, T.C., \& de Souza Braga, F.M. (2009). Desempenho de três linhagens de tilápia submetidas ao tratamento com 17- $\alpha$ metiltestosterona em condições ambientais não controladas. Revista Brasileira de Zootecnia 38:6. p. 973-979. https://doi.org/10.1590/S151635982009000600001

- Noga, E.J. (2010). Fish diseases: diagnosis and treatment. John Wiley \& Sons.

- Núñez, J., \& Duponchelle, F. (2009). Towards a universal scale to assess sexual maturation and related life history traits in oviparous teleost fishes. Fish Physiology and Biochemistry 35(1):167-80. https://doi.org/10.1007/s10695-008-9241-2

- Paul-Prasanth, B; Bhandari, R.K., Kobayashi, T., Horiguchi, R., Kobayashi, Y., Nakamoto, M., Shibata, Y., Sakai, F., Nakamura, M., \& Nagahama, M. (2013). Estrogen oversees the 
maintenance of the female genetic program in terminally differentiated gonochorists. Scientific Reports 3: 2862. https://doi.org/10.1038/srep02862

- Phelps, R.P., \& Popma, T.J. (2000). Sex reversal of tilapia. Costa-Pierce B.A.; Rakocy, J.E. Tilapia Aquaculture in the Americas 2 p. 34-59. The World Aquaculture Society, Baton Rouge, Louisiana, United States.

- Phelps, R. (2006). Hormone Manipulation of sex. Webster, CD; Lim, C (eds.). Tilapia: Biology, Culture, and Nutrition Capitulo 6 p 236. CRC Press, New York, 704 p.

- Phelps, R., \& Okoko, M. (2011). A nonparadoxical dose response to $17 \alpha-$ methyltestosterone by Nile tilapia Oreochromis niloticus (L.): Effects on the sex ratio, growth and gonadal development. Aquaculture Research. 42: $549-558$ https://doi.org/10.1111/j.13652109.2010.02650.x

- Piferrer, F. (2001). Endocrine sex control strategies for the feminization of teleost fish. Aquaculture 197:1-4 p. 229-281. https://doi.org/10.1016/S0044-8486(01)00589-0

- Popma T., \& Green B. (1990). Reversión sexual de tilapias en lagunas de tierra. International Center for Aquaculture. Alabama Agricultural Experiment Station Auburn University US. Research and Development Series $\mathrm{N}^{\circ} 35.30 \mathrm{p}$.

- Rebouças, P.M., Rocha, R. S., Silva, M.C., Barbosa-Filho, J.A.D., Farias, WR, Pinto C.R.S., \& Henrique J.C. (2014). Influence of environmental color on zootechnical performance and feeding behavior during masculinization of Nile tilapia. Journal of Animal Behaviour and Biometeorology 2: 126-130. http://dx.doi.org/10.14269/23181265/jabb.v2n4p126-130

- Rima, N.N., Rahman, M.M., \& Sarker, M.J.

(2017). Optimization of 17-alpha Methyltestosterone (MT) Hormone Dose during Masculinization of Nile Tilapia (Oreochromis niloticus) fry. Journal of Noakhali Science and Technology University, 1: 35-41

- Rivero-Wendt, C., Miranda-Vilela, A. L., Domingues, I., Oliveira, R., Monteiro, M. S., Moura-Mello, M., Matias, R., Soares, A., \& Grisolia, C. K. (2020). Steroid androgen 17 alpha methyltestosterone used in fish farming induces biochemical alterations in zebrafish adults. Journal of environmental science and health. Part A, Toxic/hazardous substances \& environmental engineering, 55(11), $1321-1332$. https://doi.org/10.1080/10934529.2020.1790954

- Sanches, L. E. F., \& Hayashi, C. (2008). Densidade de estocagem no desempenho de larvas de tilápiado-Nilo (Oreochromis niloticus L.), durante a reversão sexual. Acta Scientiarum. Animal Sciences, 21, 619-625. https://doi.org/10.4025/actascianimsci.v21i0.4299

- Shen, Z.G., Fan, Q.X., Yan, W., Zhang, Y.L., \& Wang, H.P. (2015). Effects of $17 \alpha-$ Methyltestosterone and Aromatase Inhibitor Letrozole on Sex Reversal, Gonadal Structure, and Growth in Yellow Catfish Pelteobagrus fulvidraco. The Biological Bulletin 228:2, 108-117 https://doi.org/10.1086/BBLv228n2p108

- Singh, A., \& Srivastava, P.P. (2014). A CYP19 Based Sex Determination and Monosex Production in Aquaculture Species Oreochromis niloticus L. and a Cyprinid Cyprinus carpio L. Fisheries and Aquaculture Journal, 6, 1-6. 10.4172/21503508.1000112

- Soltan, M., Hassaan, M., El-Nagaar, G., Mohammed, W., Abdelhamid1, A., El-Barbary, M., Mabrouk, E. (2013). Effect of rearing temperature and hormone treatment on sex ratio, survival and body weight of Oreochromis niloticus fry. Egyptian Journal of Aquatic Biology and Fisheries, 17(4), 13-23. https://doi.org/10.21608/ejabf.2013.2182.

- Sun, L. N., Jiang, X. L., Xie, Q.P., Yuan, J., Huang, B.F., Tao, W.J., \& Wang, D.S. (2014). Transdifferentiation of differentiated ovary into functional testis by long-term treatment of aromatase inhibitor in Nile tilapia. Endocrinology, 155(4), 1476-1488. https://doi.org/10.1210/en.2013-1959

- Tachibana, L., Leonardo, A., Corrêa, C., \& Saes, L. (2018). Stocking density of nile tilapia (Oreochromis niloticus) fry during sex reversal phase. Boletim Do Instituto De Pesca, 34(4), 483 488.

https://www.pesca.agricultura.sp.gov.br/boletim/i ndex.php/bip/article/view/817

- Thanasupsin, S. P., Chheang, L., \& Math, C. (2021). Ecological risk of $17 \alpha$-methyltestosterone contaminated water discharged from a full water 
recirculating earthen masculinization pond, Human and Ecological Risk Assessment: An International Journal, 27:6, 1696-1714. https://doi.org/10.1080/10807039.2021.1871845

- Teichert-Coddington, D., Manning, B., Eya, J. \& Brock, D. (2000). Concentration of $17 \alpha-$ Methyltestosterone in HormoneTreated Feed: Effects of Analytical Technique, Fabrication, and Storage Temperature. Journal of the World Aquaculture Society, 31: 4250. https://doi.org/10.1111/j.17497345.2000.tb00696.x

- Trejo-Quezada, A., Calzada-Ruiz, D., SorianoLuis, F., Valenzuela-Jimenez, N., Ramírez-Ochoa, M., Moreno-de la Torre, R., \& Alcántar-Vázquez, J. P. (2021). Evaluación del periodo de masculinización en la tilapia del Nilo var spring empleando 17 $\alpha$-metiltestosterona: Periodo de hormonado en tilapia. Ecosistemas y Recursos Agropecuarios, 8(1). https://doi.org/10.19136/era.a8n1.2739
- Wassermann, G. J., \& Bertolla L. O. (2002). Validation of the aceto-carmine technique for evaluating phenotypic sex in nile tilapia (Oreochromis niloticus) fry. Ciência Rural, Santa Maria 32(1):

133-139.

https://doi.org/10.1590/S010384782002000100023.

- Xu, G., Huang, T., Gu, W., Liu, E., \& Wang, B. (2021), Effects of letrozole and $17 \alpha$ methyltestosterone on gonadal development in allfemale triploid rainbow trout (Oncorhynchus mykiss). Aquaculture Research, 52: 24602469. https://doi.org/10.1111/are.15095

- Zanardi, M.F., Koberstein,T.C., Urbinati,E.C., Fagundes, M., dos Santos, M., \& Mataqueiro, M.I. (2011). Concentrações de hormônio na carcaça de tilápias-do-nilo e maturação precoce após reversão sexual. Revista Brasileira de Zootecnia, 40:1 pp. 711. https://doi.org/10.1590/S151635982011000100002 . 